\title{
Efficient technique for measuring laser frequency stability
}

\author{
A. Sargsyan ${ }^{1}$, A.V. Papoyan ${ }^{1, a}$, D. Sarkisyan ${ }^{1}$, and A. Weis ${ }^{2}$ \\ 1 Institute for Physical Research, NAS of Armenia, Ashtarak-2 0203, Armenia \\ 2 Département de Physique, Université de Fribourg, Chemin du Musée 3, 1700 Fribourg, Switzerland
}

\begin{abstract}
We propose a new technique for measuring the frequency stability of cw laser radiation. The technique relies on using the laser to be tested as a coupling laser in a scheme of electromagnetically-induced transparency (EIT), either on a $\Lambda$ or a ladder system. A second, frequency tunable laser (stabilization of this laser is not needed) is used both to act as the EIT probe laser, and to form an atomic frequency reference spectrum. The frequency stability is monitored via the frequency deviation of the EIT resonance with respect to an atomic reference frequency. We also present an improved dichroic atomic vapor laser lock (DAVLL) method for frequency stabilization based on a nanocell with a $\lambda / 2$ thickness.
\end{abstract}

PACS. 32.80.Qk Coherent control of atomic interactions with photons - 42.50.Gy Effects of atomic coherence on propagation, absorption, and amplification of light; electromagnetically induced transparency and absorption - 42.60.Lh Efficiency, stability, gain, and other operational parameters - 42.55.Px Semiconductor lasers; laser diodes

\section{Introduction}

Continuous-wave single-frequency tunable diode lasers (DL) are widely used as convenient tools in atomic physics experiments, in particular for the high-resolution spectroscopy of dilute atomic media, such as alkali vapors and beams. The transitions from the $n \mathrm{~S}_{1 / 2}$ ground state to the lowest-lying $n \mathrm{P}_{1 / 2}$ and $n \mathrm{P}_{3 / 2}$ states $\left(D_{1}, D_{2}\right.$ lines $)$ of those elements remain in the center of interest due to the simplicity of their level scheme and their large oscillator strengths. The DL emission frequency strongly depends on its temperature, injection current, external optical cavity length, and other parameters. Conventional techniques used for the active stabilization of those parameters yield a substantial reduction of the short-term noise and longterm drift of the laser frequency. A most efficient way to reduce frequency fluctuations is the locking of laser frequency to a reference frequency with an intrinsically high stability, such as an ultrastable high-finesse Fabry-Pérot, or, better, a narrow atomic resonance transition. In the past decades, many different experimental schemes were elaborated for locking DL frequencies to atomic resonance lines, such as saturated absorption [1,2], magneto-optical processes [3], including the technique of dichroic atomic vapor laser locking (DAVLL) [4-7], transversely-pumped thin cell spectroscopy [8], polarization spectroscopy [9], and selective reflection spectroscopy [10-13].

Recently a new spectroscopic technique was developed that uses vapor cells of sub-micrometer-range thickness

\footnotetext{
${ }^{a}$ e-mail: papoyan@ipr.sci.am
}

(so called nanocells, or extremely thin cells, etc.). The interaction of atoms with laser radiation in such cells is strongly anisotropic [14-17]. Atoms with a non-zero longitudinal (with respect to the laser beam) velocity component experience frequent quenching collisions with the cell walls (windows). As a result, the interaction is favored for atoms flying parallel to the cell walls, i.e., perpendicular to the laser beam. Such atoms yield a nearly Doppler-free response. This key feature, together with a Dicke-type of coherent spectral narrowing occurring for a vapor thicknesses $L=(2 n+1) \lambda / 2$ and a Fabry-Pérot type of behavior occurring for $L=n \lambda / 2$ [15-17], where $n$ is an integer, determine the significant difference of the optical spectra recorded with such nanocells from those in ordinary, cmsized cells.

In the present work, we have modified the DAVLL method by use of a Rb nanocell with a thickness $L=\lambda / 2$, which yields error signals with a much steeper slope than obtained in ordinary cm-sized cells.

Besides the frequency locking proper, an important issue is the quantitative determination of the characteristics and quality of the lock. A conventional procedure for characterizing the stabilization of a DL implies the recording and analysis of a beat-note between the laser of interest and an auxiliary well-stabilized laser. Here we present a novel, simple and robust technique for testing the laser frequency stabilization. The technique uses an electromagnetically-induced transparency (EIT) resonance (see review [18]) formed by the laser to be tested (coupling laser) and an auxiliary frequency tunable laser 
(probe laser). The EIT process can be realized either on a $\Lambda$ or a ladder system. The probe laser also serves to form an atomic reference spectrum. It is important to note that the probe laser does not need to be frequency stabilized. The frequency stability is measured by monitoring the deviation of the EIT resonance frequency with respect to the reference frequency. The benefit of the proposed scheme is the fact that it does neither require a highly-stable reference laser, nor any complex electronics.

\section{Experimental details}

The design of the nanocells used to realize the modified DAVLL technique for laser frequency locking and for forming the reference spectrum, is similar to the one described in [14]. The windows (30 $\mathrm{mm}$ diameter, $3 \mathrm{~mm}$ thick) are made from very well polished wafers of garnet (YAG), which is resistant to the highly corrosive hot alkali vapor. The roughness of the inner surfaces of the YAG windows is on the order of $\lambda / 10$. In order to realize a verticallywedged gap between the YAG windows allowing a smooth variation of the cell thickness, a $1 \mathrm{~mm}$ wide and $10 \mathrm{~mm}$ long $\mathrm{Al}_{2} \mathrm{O}_{3}$ strip coating with a thickness of approximately $1000 \mathrm{~nm}$ was deposited near the lower edge of the surface of one of the windows. A sapphire sidearm with a "molybdenum glass" termination is used for filling the cell with $\mathrm{Rb}$ in the same way as for all-glass cells. A hole is drilled in the bottom of the YAG windows for attaching the sidearm. Before filling the nanocell with natural rubidium, the cell was carefully outgassed during $8 \mathrm{~h}$ at $400{ }^{\circ} \mathrm{C}$. The wedged gap between the windows allows us to vary the thickness of the vapor column traversed by the laser beam by a vertical translation of the cell, and to adjust the thickness to be $L=\lambda / 2$ or $L=\lambda$, which is important for achieving the narrowest sub-Doppler linewidth [15,19], or for the formation of the reference spectrum [20]. An interferometric mapping of the gap thickness was performed using a He-Ne laser, showing that the thickness of the gap varies between $100 \mathrm{~nm}$ and $1000 \mathrm{~nm}$. The upper temperature limit for the nanocell is $\sim 500{ }^{\circ} \mathrm{C}$. The nanocell is operated with a specially designed two-chamber oven, which enables the independent temperature control of the cell body and sidearm. The atomic vapor density is determined by the temperature of the vapor-liquid metal interface in the sidearm. The sidearm temperature was typically kept at $110^{\circ} \mathrm{C}$, while the window temperature was set to a somewhat higher value $\left(120{ }^{\circ} \mathrm{C}\right)$ in order to prevent metal condensation. The corresponding atomic number density is $N_{\mathrm{Rb}} \approx 10^{13} \mathrm{~cm}^{-3}$, and the collisionally broadened width of the $D_{2}$ line is $\approx 2 \mathrm{MHz}$. The thin cell of sub-millimeter thickness used for recording the nanocell resonance was of similar design.

A schematic diagram of the optical part of the experimental setup is shown in Figure 1. Tunable free-running single-mode laser diodes (Sanyo model DL-7140-201W) with a maximum power of $70 \mathrm{~mW}$ and a spectral linewidth $\Delta \nu_{L}<5 \mathrm{MHz}$, emitting in the region of $\mathrm{Rb} D_{2}$ line, were used to form the coupling and probe beams, respectively. After passing through Faraday isolators (FI), both beams

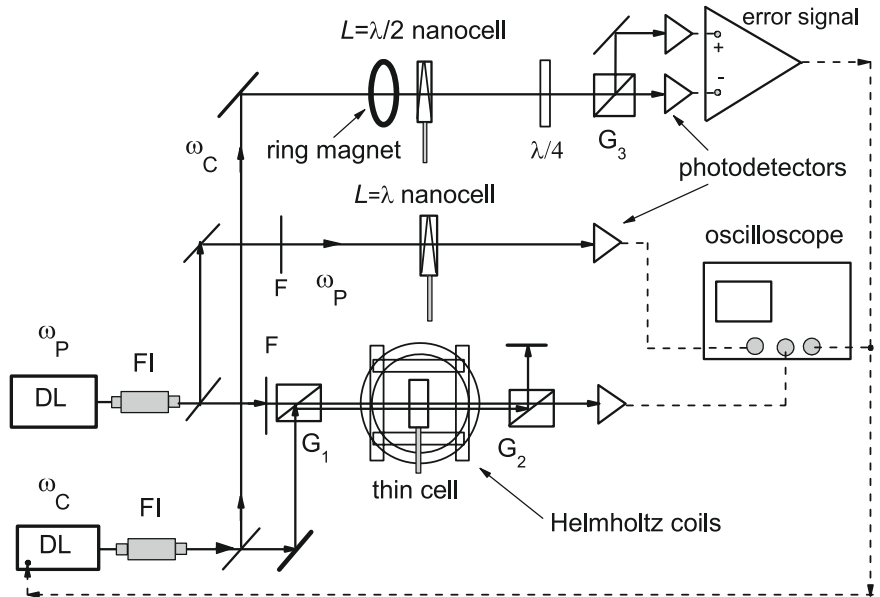

Fig. 1. Experimental setup for stabilization and testing of laser diode frequency. DL: diode laser, FI: Faraday isolator, G: Glan prisms.

(3 mm diameter) were well superposed and directed with the help of a first Glan prism $\left(\mathrm{G}_{1}\right)$ onto the thin cell with a thickness $L=0.7 \mathrm{~mm}$ at near-normal incidence. In this way the coupling and probe beams have mutually orthogonal linear polarizations. A second Glan prism $\left(\mathrm{G}_{2}\right)$ was used for separating the coupling and probe beams, so that only the probe beam transmission could be monitored. The thin cell was placed inside three pairs of mutually perpendicular Helmholtz coils providing the possibility to cancel the laboratory magnetic field, or to apply a homogeneous magnetic field, when needed. The power of the beams was recorded by photodiodes followed by operational amplifiers; and the photodiode signals were stored in a two-channel digital storage oscilloscope (Tektronix TDS 3032B). Neutral density filters (F) were used to adjust the power of the individual beams.

The formation of a frequency reference spectrum was realized with the help of an auxiliary nanocell. When the nanocell thickness is $L \approx \lambda$, sub-Doppler peaks of reduced absorption appear in its transmission spectrum. These peaks are centered on the individual hyperfine transitions [20] as in saturated absorption. Unlike in saturated absorption spectroscopy, however, the nanocell technique shows no crossover resonances.

\section{Locking the laser radiation frequency by DAVLL technique using a nanocell}

The primary passive stabilization of the laser frequency was realized by stabilizing the LD temperature (accuracy $0.01{ }^{\circ} \mathrm{C}$ ) and its injection current (5 $\mu \mathrm{A}$ accuracy). An improved DAVLL method [4,5], realized using a separate $L=\lambda / 2$ nanocell, was implemented for locking the coupling laser frequency to an atomic resonance line. The idea of improvement is as follows. The efficiency of the frequency lock strongly depends on the slope of the error signal [13]. In a cm-sized cell, such as used with the conventional DAVLL method, the error signal $[4,5]$ 


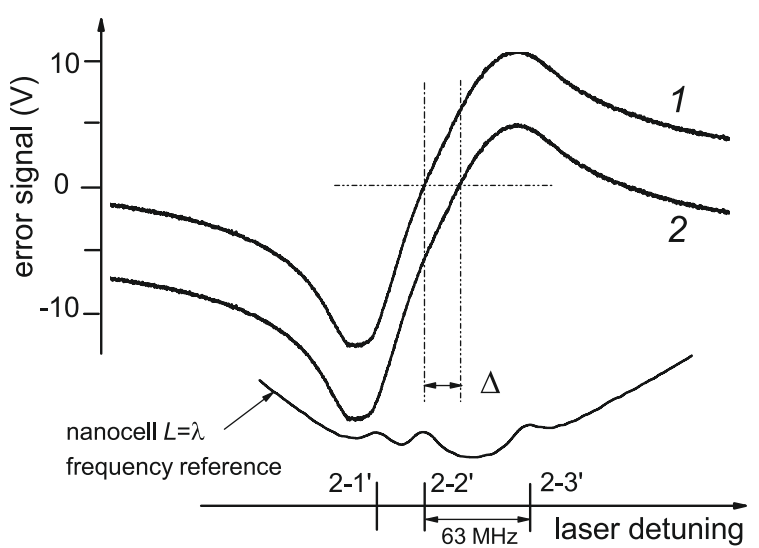

Fig. 2. Curves 1 and 2: error signal spectra for different offset voltages; lower curve: reference spectrum of the $L=\lambda$ nanocell.

is Doppler-broadened. The sub-Doppler resonances occurring in a $L=\lambda / 2$ nanocell are approximately 4 times narrower [19], and the slope of the error signal increases accordingly.

The linearly-polarized laser beam propagates through the nanocell placed in an external longitudinal magnetic field. Due to the Zeeman shifts of the atomic sublevels, the absorption spectra of the $\sigma^{-}$and $\sigma^{+}$components of the linearly polarized light shift in opposite directions. A $\lambda / 4$ plate placed after the cell transforms the $\sigma^{-}$and $\sigma^{+}$ components into mutually orthogonal linear polarizations, which are spatially separated by a Glan prism $\left(\mathrm{G}_{3}\right)$ and detected by two photodiodes, as shown in the upper right corner of Figure 1. The error signal (shown in Fig. 2) is obtained by the electronic subtraction of the photodiode signals, which yields a dispersive resonance. Frequencycontrolling feedback to the laser is achieved by controlling the laser's injection current. A longitudinal magnetic field is provided by a permanent ring magnet having a $2 \mathrm{~mm}$ diameter bore for passing the laser beam. The shape of the error signal spectrum depends on the sign and magnitude of the magnetic field. The magnetic field strength (measured by a calibrated Hall probe) is controlled by a longitudinal motion of the ring magnet through a micrometer translation stage. The ring magnet produces a strongly inhomogeneous field, which varies, however, only by a few $\mu \mathrm{T}$ inside of the atomic vapor column because of its small thickness $(780 \mathrm{~nm})$ [21]. The possibility to substitute Helmholtz coils by a permanent magnet is an additional asset of the error signal formation in nanocells.

Typical error signal spectra of the $F_{g}=2 \rightarrow F_{e}=$ $1,2,3$ transitions of the ${ }^{85} \mathrm{Rb} D_{2}$ line are presented in Figure 2. In order to increase the absorption in the nanocell, the side-arm temperature was kept at $130{ }^{\circ} \mathrm{C}$ (with a 20 degrees higher window temperature). The lower curve in the figure is a frequency reference spectrum formed by the nanocell with $L=\lambda$ thickness [20]. The feedback is adjusted to lock the signal to zero voltage. By adding an offset voltage (horizontal dashed line in Fig. 2) to the error signal one can thus control the (frequency) position of the lock point (vertical dashed line). The error signal spectra in Figure 2 confirm the expected spectral narrowing and

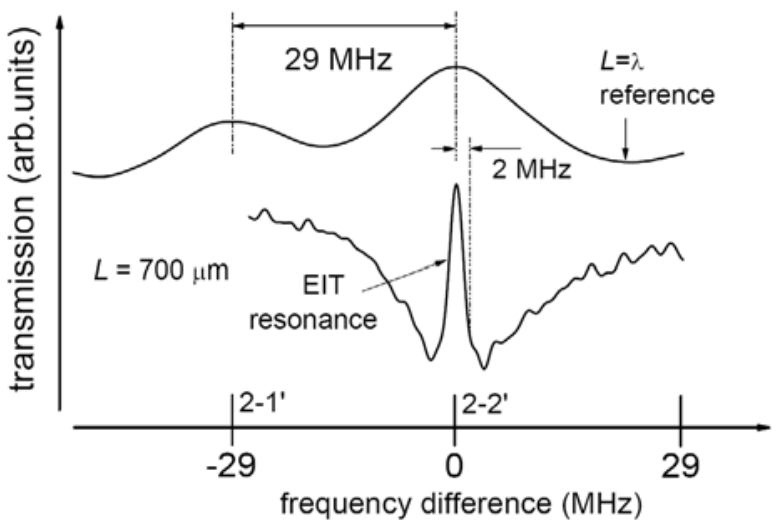

Fig. 3. Lower curve: transmission spectrum of the probe laser with EIT resonance; upper curve: frequency reference spectrum formed by the probe laser in a $L=\lambda$ nanocell.

the substantial increase of the slope steepness compared to a Doppler-broadend signal in an ordinary cell. The error signal represented by curve 1 allows locking the laser diode frequency to the $5 \mathrm{~S}_{1 / 2}, F_{g}=2 \rightarrow 5 P_{3 / 2}, F_{e}=2$ transition, while the signal of curve 2 corresponds to a $30 \mathrm{MHz}$ blue-shifted locking frequency.

\section{EIT technique for measuring frequency stability}

The idea of the proposed technique for the quantitative characterization of laser frequency stability, in particular the determination of a residual frequency drift, is to use the laser under investigation as a coupling laser for producing a dark EIT resonance. A second frequency-tunable laser was used as probe laser, as well as for forming a frequency reference spectrum. By monitoring the difference of the EIT resonance position and the position of the atomic frequency reference peak in the $L=\lambda$ nanocell, one has a direct measure of the locking efficiency and accuracy. It is noteworthy that the probe laser does not need to be frequency-stabilized, as explained below.

When the coupling laser is locked to the $F_{g}=3 \rightarrow$ $F_{e}=2$ transition, and the probe laser is scanned across the $F_{g}=2 \rightarrow F_{e}=1,2,3$ transitions (Fig. 4), a $\Lambda$ system is realized when the probe frequency is resonant with the $F_{g}=2 \rightarrow F_{e}=2$ transition. The corresponding dark resonance is shown as lower curve in Figure 3 . The power of the coupling and probe lasers were 0.3 and $0.1 \mathrm{~mW}$, respectively. Note that this sharp resonance can be formed by coherent population trapping $(\mathrm{CPT})$ process as well. The cell thickness was $L=0.7 \mathrm{~mm}$, and the sidearm temperature was $70^{\circ} \mathrm{C}$. Dark resonance formation in thin cells was recently presented in $[22,23]$. It was shown that a decrease of the cell thickness down to $L=0.7 \mathrm{~mm}$ does not cause noticeable degrading of the dark resonance width and contrast (for signal/noise reasons the use of cm-sized cells for dark resonance studies may be preferable). The upper curve of Figure 3 is the reference spectrum, in which 


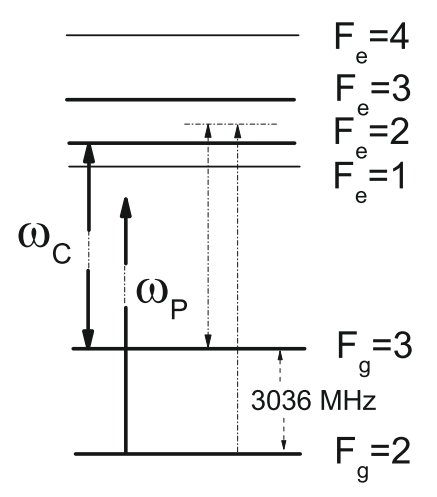

Fig. 4. Energy levels of ${ }^{85} \mathrm{Rb}$; the coupling laser is in resonance with the $F_{g}=3 \rightarrow F_{e}=2$ transition, and the probe laser is scanned across the $F_{g}=2 \rightarrow F_{e}=1,2,3$ transition.

velocity selective optical pumping/saturation (VSOP) resonances, located at the $F_{g}=2 \rightarrow F_{e}=1,2$ transitions in the transmission spectrum of the nanocell with $L=\lambda$, are well seen. For this configuration of coupling and probe lasers frequencies, the EIT resonance is located exactly at the position of $F_{g}=2 \rightarrow F_{e}=2 \mathrm{VSOP}$ resonance (lower curve in Fig. 3).

If the coupling (tested) laser frequency is blue-shifted by, say $2 \mathrm{MHz}$, the EIT condition will only be fulfilled when the probe frequency, $\nu_{P}$, has an identical blue shift, corresponding to a new $\Lambda$ system as indicated by the dotted lines in Figure 4. In consequence, the EIT resonance position will be blue-shifted by the same amount (vertical dotted line on the right of Fig. 3). This shift will be well detectable in the spectra presented in Figure 3. It is important to note that any frequency drift of the free running second (probe) laser will cause an overall simultaneous shift of both spectra in Figure 3, while the difference between the EIT and VSOP resonance frequencies will not be affected. Figure 5 presents the frequency deviation of the dark resonance position with respect to the $F_{g}=2 \rightarrow F_{e}=2 \mathrm{VSOP}$ frequency as a function of time. The proposed stabilization testing scheme is thus capable of detecting deviations of the coupling laser frequency exceeding $200 \mathrm{kHz}$. Note that the EIT resonance lineshape is shown to be insusceptible to $\pm 10 \mathrm{MHz}$ frequency detuning of the coupling laser from $F_{g}=3 \rightarrow F_{e}=2$ transition.

The recorded limit of frequency resolution is sufficient for many applications, in particular, in atomic physics (note that even for the conditions of Fig. 3, the proposed technique is much more accurate than the one presented in [13]). If needed, the accuracy can be increased by optimization of experimental conditions. As is seen from Figure 3, the frequency resolution depends both on the linewidth of EIT resonance and the sharpness of frequency reference spectrum formed by the probe laser. It is well known that the EIT resonance linewidth can be strongly reduced [18]. Also, the width of reference line formed by the probe laser can be reduced $(\sim 5$ times $)$ with the use of saturated absorption spectrum in a small external magnetic field [24]. This optimization, as well as evaluation of frequency stability by standard means (statistical uncer-

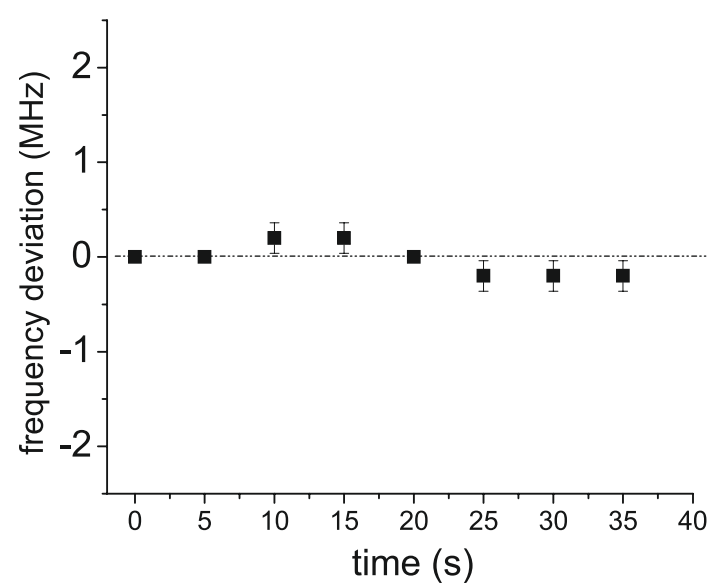

Fig. 5. Deviation of dark resonance frequency with respect to VSOP resonance frequency as a function of time.

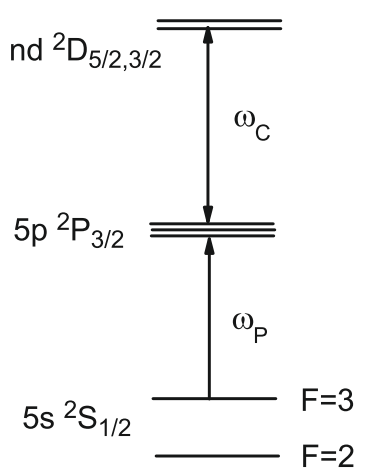

Fig. 6. Ladder $(\Xi)$ system for dark resonance formation.

tainty, Allan variance, noise budget, etc.) was out of the scope of present paper, which solely presents a technique that can be further elaborated.

The suggested technique for testing the EIT resonance frequency position (TEITR) can equally exploit EIT in ordinary cm-size cells, not only in $\Lambda$ but also in ladder ( $\Xi$ ) systems (see Fig. 6). In [25], an efficient EIT involving Rydberg levels of $\mathrm{Rb}$ was demonstrated. The coupling laser wavelength was in the range of $479-484 \mathrm{~nm}$, resonant with $5 \mathrm{P}_{3 / 2} \rightarrow n \mathrm{D}$, where $n>20$ is integer, while the tunable probe laser wavelength $(\approx 780 \mathrm{~nm})$ was scanned across $5 \mathrm{~S}_{1 / 2} \rightarrow 5 \mathrm{P}_{3 / 2}$ as shown in Figure 6 [25]. An efficient dark resonance can be formed also for $5 \mathrm{D}$ state, when the coupling wavelength is $776 \mathrm{~nm}$ [26]. Spectra similar to those presented in Figure 3 can be obtained for $\Xi$ systems as well, thus allowing the use of TEITR for testing laser frequency stabilization also in the $479-484 \mathrm{~nm}$ wavelength range. In addition, there is a large number of $\Lambda$ and $\Xi$ systems with probe laser wavelengths of $766,770,780$, 794,852 , or $894 \mathrm{~nm}\left(D_{2}\right.$ and $D_{1}$ lines of $\mathrm{K}, \mathrm{Rb}$, and $\mathrm{Cs}$ atoms), at which single-frequency DLs are available, and the coupling laser wavelength can be chosen in the range of $480 \div 900 \mathrm{~nm}$. Thus, TEITR can be useful for testing the laser frequency stabilization in a wide wavelength range. 


\section{Conclusions}

A new reliable method for testing laser diode frequency stabilization, TEITR, was developed. It is based on the effect of electromagnetically induced transparency (EIT). With this technique the tested laser is used as a coupling laser for the formation of the EIT resonance. A second frequency-tunable laser, for which no stabilization is required, is used as a probe laser (and also for forming the frequency reference spectrum). By monitoring the frequency position of the EIT resonance with respect to the atomic reference frequency, it is easy to monitor deviations of the laser frequency. Using different $\Lambda$ and $\Xi$ systems, it is possible to implement the TEITR technique for measuring the frequency stability of lasers with wavelengths in the range of 480-900 $\mathrm{nm}$.

We have experimentally demonstrated the efficiency of the proposed TEITR method for the case when the coupling laser frequency was stabilized using an improved DAVLL method, based on a Rb nanocell with a thickness $L=\lambda / 2$. This locking scheme allows the formation of an error signal with a much steeper slope compared to the one realized using ordinary $\mathrm{cm}$-thick vapor cells.

The authors are grateful to A.S. Sarkisyan for his valuable participation in fabrication of the nanocells. This work was supported, in part, by Swiss SCOPES Grant IB7320-110684/1 and by INTAS South-Caucasus Grant 06-1000017-9001.

\section{References}

1. H. Hori, Y. Kitayama, M. Kitano, T. Yabuzaki, T. Ogawa, IEEE J. Quant. Electron. 19, 69 (1983)

2. Y. Shevy, H. Deng, Opt. Lett. 23, 472 (1998)

3. A. Weis, S. Derler, Appl. Opt. 27, 301 (1988)

4. K.L. Corwin, Z.-T. Lu, C.F. Hand, R.J. Epstein, C.E. Wieman, Appl. Opt. 37, 3295 (1998)

5. V.V. Yashchuk, D. Budker, J.R. Davis, Rev. Sci. Instrum. 71, 341 (2000)

6. M.A. Clifford, G.P.T. Lancaster, R.S. Conroy, K. Dholakia, J. Mod. Opt. 47, 1933 (2000)

7. G. Wasik, W. Gawlik, J. Zachorowski, W. Zawadzki, Appl. Phys. B 75, 613 (2002)
8. K. Fukuda, N. Furukawa, S. Hayashi, M. Tachikawa, IEEE Trans. Ultrason. Ferroelectr. Freq. Control 47, 502 (2000)

9. A. Weis, V.A. Sautenkov, T.W. Hänsch, J. Phys. II (France) 3, 263 (1993)

10. R. Loe-Mie, A.V. Papoyan, A.M. Akulshin, A. Lezama, J.R. Rios Leite, O. Lopez, D. Bloch, M. Ducloy, Opt. Commun. 139, 55 (1997)

11. R. Müller, A. Weis, Appl. Phys. B 66, 323 (1998)

12. R.N. Li, S.T. Jia, D. Bloch, M. Ducloy, Opt. Commun. 146, 186 (1998)

13. E.A. Gazazyan, A.V. Papoyan, D. Sarkisyan, A. Weis, Laser Phys. Lett. 4, 801 (2007)

14. D. Sarkisyan, D. Bloch, A. Papoyan, M. Ducloy, Opt. Commun. 200, 201 (2001)

15. G. Dutier, A. Yarovitski, S. Saltiel, A. Papoyan, D. Sarkisyan, D. Bloch, M. Ducloy, Europhys. Lett. 63, 35 (2003)

16. D. Sarkisyan, T. Varzhapetyan, A. Sarkisyan, Yu. Malakyan, A. Papoyan, A. Lezama, D. Bloch, M. Ducloy, Phys. Rev. A 69, 065802 (2004)

17. C. Andreeva, S. Cartaleva, L. Petrov, S.M. Saltiel, D. Sarkisyan, T. Varzhapetyan, D. Bloch, M. Ducloy, Phys. Rev. A 76, 013837 (2007)

18. M. Fleischhauer, A. Imamoglu, J.P. Marangos, Rev. Mod. Phys. 77, 633 (2005)

19. A. Sargsyan, D. Sarkisyan, Y. Pashayan-Leroy, C. Leroy, P. Moroshkin, A. Weis, J. Contemporary Phys. 43, 7 (2008)

20. A. Sargsyan, D. Sarkisyan, A. Papoyan, Y. PashayanLeroy, P. Moroshkin, A. Weis, A. Khanbekyan, E. Mariotti, L. Moi, Laser Phys. 18, 749 (2008)

21. A. Sargsyan, G. Hakhumyan, A. Papoyan, D. Sarkisyan, A. Atvars, M. Auzinsh, Appl. Phys. Lett. 93, 021119 (2008)

22. Y. Pashayan-Leroy, C. Leroy, A. Sargsyan, A. Papoyan, D. Sarkisyan, J. Opt. Soc. Am. B 24, 829 (2007)

23. D. Sarkisyan, A. Sargsyan, A. Papoyan, Y. PashayanLeroy, Proc. SPIE 6604, 660405 (2007)

24. R.Kh. Drampyan, A.D. Greentree, A.V. Durrant, in Optics Research Trends, edited by P. Gallico (Nova Science Publishers, 2007), p. 271

25. A.K. Mohapatra, T.R. Jackson, C.S. Adams, Phys. Rev. Lett. 98, 113003 (2007)

26. J. Gea-Banacloche, Y.-Q. Li, S.-Z. Jin, M. Xiao, Phys. Rev. A 51, 576 (1995) 